\title{
LA LOGÍSTICA COMO ESTRATEGIA PARA PROVEER DE INTELIGENCIA A LAS ORGANIZACIONES
}

\section{LOGISTICS AS A STRATEGY TO PROVIDE INTELLI- GENCE ORGANIZATIONS}

\section{RESUMEN}

En el presente documento se expone una estrategia para disminuir el fracaso que existe en el proceso de apropiación del conocimiento para soportar la toma de decisiones en organizaciones de producción de bienes y servicios.

Se evidencia la falta de hacer efectivo el conocimiento para obrar y tomar decisiones, es decir no se potencia el uso del conocimiento por deficiencia en el sistema logístico requerido para su apropiación.

Se busca mostrar un camino para hacer que las organizaciones se provean de inteligencia por lo que se propone la utilización de un sistema de aprendizaje organizacional, basado en el diseño de un operador de integración logística de conocimiento construido a partir de la unión sinérgica de sistemas de gestión de conocimiento y de sistemas de soporte a la decisión.

La estrategia para construir el estado del arte parte del concepto propio de inteligencia individual, y por interacción como afecta a las estructuras organizacionales, para luego abordar los conceptos de inteligencia de negocios, inteligencia organizacional y gestión de conocimiento y su papel en el proceso de aprendizaje organizacional.

Por lo que la hipótesis de la propuesta es:

"Con la Integración Logística de Conocimiento, que se conFigura como la unión sinérgica de un sistema de soporte a la decisión (DSS) y la gestión del flujo de conocimiento; se dota de inteligencia a las organizaciones de producción de bienes y servicios, con capacidad para actuar exitosamente frente a procesos de toma de decisiones que no son abordados con las teorías y enfoques tradicionales."

Para lo cual en primer lugar se propone determinar el estado del arte a partir de la caracterización de la estructura y el ambiente organizacional, determinando los diferentes factores y elementos, en donde se presentan problemas de decisión en la gestión de organizaciones productivas de bienes y servicios en ambientes estratégicos, tácticos y operativos y que proporcione inteligencia organizacional.

\section{César Amílcar López Bello}

Ingeniero Industrial - Universidad Distrital "Francisco José De Caldas"Colombia. Maestría En Ingeniería Industrial - Universidad De Los Andes - Colombia.

Docente - Universidad Distrital "Francisco José De Caldas" - Colombia clopezb@udistrital.edu.co, msccesar. lopez@gmail.com

\section{Sandro Javier Bolaños Castro}

Ingeniero de Sistemas - Universidad Distrital "Francisco José De Caldas" Colombia. Doctorado en Informática - Universidad Pontificia de Salamanca- España.

Coordinador Especializaciones Ingeniería de Software - Universidad Distrital "Francisco José De Caldas" - Colombia - sbolanos@udistrital. edu.co

German Andrés Méndez Giraldo Ingeniero Industrial -Universidad Distrital "Francisco José De Caldas" - Colombia. Doctorado En Ciencias Técnicas - Universidad Central de Las Villas - Cuba.

Docente líder del grupo de investigación CES- Universidad Distrital "Francisco José De Caldas"- Colombia-gmendez@udistrital.edu.co

Tipo: Artículo de revisión

Fecha de Recepción: 11 de abril de 2014 Fecha de Aceptación: Junio 22 de 2015 
Palabrasclave: Logísticadel conocimiento, Aprendizaje organizacional, inteligencia organizacional, gestión del conocimiento.

\section{ABSTRACT}

This paper presents a strategy to decrease the failure that exists in the process of appropriation of knowledge to support decision-making in organizations producing goods and services.

Failure to realize the knowledge to act and make decisions, that is not enhanced by the use of knowledge logistics system deficiency appropriation required for evidence.

It seeks to show a way for organizations to provide intelligence so the use of an organizational learning system based on the design of a logistics operator integration of knowledge built from synergistic union of systems is proposed knowledge management systems and decision support.

The strategy to build state of the art of the very concept of individual intelligence, interaction and how it affects organizational structures, and then address the concepts of business intelligence, organizational intelligence and knowledge management and its role in the learning process organizational.

So the hypothesis is proposed:

"With the Logistics Integration of Knowledge, which is conFiguraured as the synergistic union of a system of decision support systems (DSS) and managing the flow of knowledge; is endowed with intelligence organizations producing goods and services, able to act successfully against decision-making processes that are not dealt with theories and traditional approaches. "

To which first proposes determine the state of the art from the characterization of the structure and the organizational environment, determining the different factors and elements, where decision pro blems arise in the management of productive organizations of goods and services at strategic, tactical and operational intelligence and provide organizational environments.

Should clearly establish the purpose and scope of the article while shortly highlighting objectives, methods, results and major conclusions. Should not exceed 150 words

Keywords: Logistics knowledge, organizational learning, organizational intelligence, knowledge management.

\section{INTRODUCCIÓN}

\section{- DEFINICIÓN DEL PROBLEMA}

En la medida en que las organizaciones se desarrollan e interactúan con su entorno se vuelven más complejas, es así como en el proceso de gestión se presentan un sinnúmero de problemas que deben ser resueltos de manera que garanticen su sostenibilidad en un entorno dinámico y cambiante conforme a las tendencias de un mundo globalizado.

En aras de resolver los problemas complejos que conllevan dificultad en su tratamiento, es necesario orientar su solución en función de los propósitos de la organización de una manera sistemática, con personas altamente capacitadas y dotadas para usar herramientas para el análisis y tratamiento de información base para realizar procesos de toma de decisiones de una manera eficiente y efectiva propio de organizaciones inteligentes.

Las empresas tratan de adoptar filosofías y adaptarse a modelos y estructuras organizacionales conforme a las tendencias de un mundo globalizado, pero al intentar aplicar sus principios, y utilizar sus técnicas no surten el efecto esperado.

Es así como, las empresas para obtener mayor productividad y competitividad conscientemente realizan inversiones en capacitación de su personal para apropiar tecnologías y mejorar sus procesos, pero debido a diferentes razones existe una brecha en la efectividad y eficiencia del conocimiento adquirido por las personas y el apropiado para el desarrollo de 
las organizaciones.

Ahora bien, muchas empresas fracasan, pocas empresas se desarrollan y algunas se mantienen, en un mercado que cada día demuestra ser más cambiante, este carácter dinámico obedece a las exigencias, sociales, culturales, económicas, Y ello hace que las estrategias, metodologías y técnicas convencionales se queden cortas para tal reto. Entre las variables críticas están:

x_1 La respuesta a los cambios del mercado es lenta.

x_2 El individuo es un componente de la organización

$\mathrm{x} \_3$ No hay un sentido sinérgico

x_4 Si los individuos aprenden las organizaciones no

x_5 No se despliega el flujo de conocimiento adquirido hacia el desarrollo de la organización x_6 No existen parámetros ni políticas para propiciar un aprendizaje organizacional.

x_7 No se utilizan adecuadamente las herramientas y técnicas como soporte a la toma de decisiones.

x_8 No hay suficiente conocimiento del uso de técnicas y métodos para la toma de decisiones.

Es evidente que el fracaso en la creación y desarrollo de las empresas se debe al desconocimiento del comportamiento del sistema organizacional y de su entorno, ya sea la de no conocer el mercado y las preferencias de los clientes, de la falta de conocimiento de los procesos y de la tecnología, de no saber comprender el comportamiento de los proveedores y de los flujos de material, de la cultura organizacional y del impacto al medio ambiente como de observa en la Figura 1.

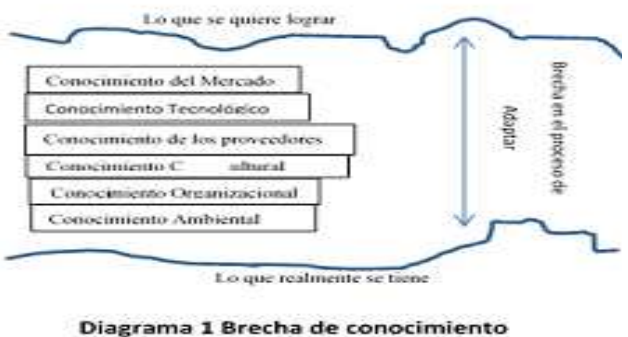

Figura 1. Grupo de conceptos

\section{ANTECEDENTES}

La construcción de sistemas de apoyo a la decisión construido en forma integral se constituye en un dispositivo que permite procesar información para la generación de conocimiento que a su vez clarifica y orienta el proceso de toma de decisiones.

El propósito de un sistema de apoyo a la decisión es proveer de herramientas para el análisis y suministrar bases (información y estructura) para la resolución de problemas de gestión de las organizaciones de producción de bienes y servicios.

Para describir el papel del proceso de toma de decisiones basado en un sistema de apoyo a la decisión DSS del inglés (Decision Support System) se propone el modelo elaborado por el presente autor en el diagrama 2.

El proceso se inicia a partir de la primera etapa que consiste en definir el sistema, en él se determinan a partir de la observación y el análisis los diferentes componentes, se detectan las relaciones e interacciones entre ellos, las entradas y diferentes insumos que alimentan el sistema productivo, se delimita su entorno inmediato con el resto del universo, se identifica su proceso de conversión, además de definir el que, el cómo, el cuándo, el porqué, el para qué y para quien se produce o se presta un servicio, se idéntica la cultura organizacional, su filosofía (bajo que principios, estrategias, objetivos y políticas), también cuáles son sus problemas, es decir se debe establecer un diagnóstico integral que represente la situación actual de la organización y su prospectiva.

La segunda etapa consiste en representar el sistema real y sus problemas en modelos, se entiende que los sistemas se pueden representar de varias maneras dando origen a diferentes modelos, donde entre ellos por una parte, se tiene complementariedad (por ejemplo un esquema, o un diagrama permiten ayudar al entendimiento de un modelo matemático abstracto) o desde otro punto de vista los modelos pueden tener el carácter de representaciones alternativas, dado que la funcionalidad del modelo depende de su manejabilidad, si el modelo es muy complejo, es posible que no se entienda, 
o no se tenga los recursos suficientes para manipularlo y por lo tanto de poca utilidad para la resolución de los problemas, o por lo contrario al buscar simplicidad no suministrar la información suficiente para soportar la toma de decisiones.

La naturaleza de los modelos que pueden ser de carácter cualitativo y cuantitativo, la selección de herramientas para el tratamiento de información y la formación y capacitación de analistas y personal altamente capacitado para enfrentar proceso de toma de decisiones, así como el conocimiento inmerso en los diferentes actores de la organización y de la misma como un todo constituyen en esencia los elementos estructurales del DSS.

La tercera etapa consiste en identificar las fuentes de generación de datos, fijar los procesos para la captura de los mismos, identificar las herramientas de análisis de estos y evaluar su validez para construir así la información de entrada para alimentar los modelos del DSS.

La cuarta etapa consiste en determinar los otros insumos que se requieren para estructurar el DSS es decir, los insumos tangibles como son los algoritmos, herramientas computacionales (Software y Hardware), así como el dimensionamiento de la capacidad requerida por los analistas y los tomadores de decisión.

Es evidente que para validar esta etapa se necesita identificar las características ideales de estos recursos para hacer factible el uso del DSS.

La quinta etapa trata del diseño del proceso de conversión de la información en el DSS es decir como experimentar y como operar para la producción del conocimiento base para la toma de decisiones (hay que fijar escenarios, y formular estrategias y alternativas de solución) La sexta etapa consiste en a partir de la información de salida evaluar la viabilidad y la validez de la información frente a las características del comportamiento del sistema real, para lo cual es necesario comprender el funcionamiento y comportamiento del sisma real y se tener la capacidad para evaluar el impacto de una decisión en la organización como un todo y de su entorno inmediato.

La séptima etapa consiste en proporcionar un conocimiento para que el tomador de decisio- nes obre con sabiduría en el proceso de toma de decisiones afectando positivamente a la organización.

Finalmente, la dinámica de alimentar el proceso de toma de decisiones basado en un DSS constituye el rasgo que caracteriza a una organización inteligente.

Ahora bien, el concepto de inteligencia organizacional difiere del concepto de inteligencia de negocios y no es simplemente una herramienta para la toma de decisiones a partir del tratamiento de información, sino se debe ver como de un conjunto de principios y elementos conceptuales que potencian a las empresas en diferentes dimensiones en todos los niveles de actuación para lo cual es se requiere proveer de capacidad a las personas para que actúen en función del crecimiento individual y colectivo, y proporcionar a la organización de un ambiente orientado hacia el logro del objetivo misional fomentando una cultura del trabajo en equipo. Se debe proveer de capacidad a la organización $\mathrm{y}$ a las personas en diferentes dimensiones para actuar exitosamente en forma integral y dinámica.

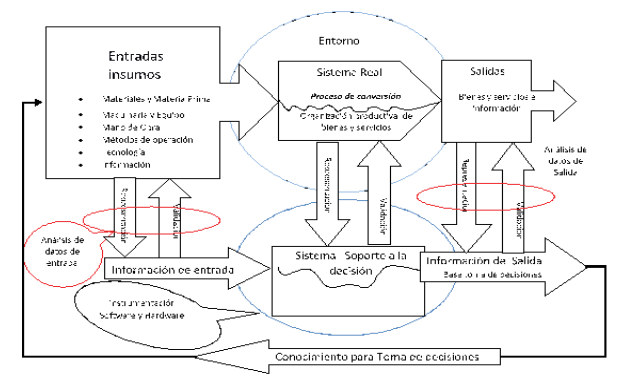

Figura 2.Sistemas de conocimiento

El propósito final consiste entonces, en desarrollar procesos metodológicos orientados a la adquisición de las capacidades que hagan a la organización ágil y rápida frente a los procesos de toma de decisiones frente a las tendencias que rige la economía globalizada y basada en la era del conocimiento.

Una perspectiva más amplia muestra como la información de salida y su validación consti- 
tuyen conocimiento que aunado con el conocimiento acumulado, la interacción con el entorno y los procesos de capacitación y formación se integran como un sistema de gestión de conocimiento para el apoyo a la toma de decisiones, como se muestra en la Figura 2.

Se entiende que para la integración del DSS y de modelos de gestión de conocimiento proporcionan una base sólida para que las organizaciones se doten de inteligencia para la toma de decisiones asertiva.

Para lograr que una organización se dote de inteligencia entonces surgen interrogantes tales como:

¿Cuál es la estructura del negocio, su capacidad y funcionalidad en espacio y tiempo?

¿Cómo afrontar los cambios y tendencias del mercado?

¿Cómo actuar en forma coordinada para que las diferentes áreas y funciones de la organización actúen sincrónicamente para el crecimiento eficiente y efectivo de la organización?

¿Cómo interactuar con los diferentes eslabones de la cadena de suministro para hacer eficiente el sistema de abastecimiento como un todo? ¿Cómo actuar en concordancia frente al impacto al medio ambiente?¿Cómo crear una infraestructura de información para el tratamiento de problemas y la toma de decisiones en estados de complejidad? Basados entonces en todos estos cuestionamientos y desplegando los principios expuestos en las diferentes teorías sobre inteligencia individual se puede estructurar conceptualmente la inteligencia organizacional en diferentes dimensiones.

\section{INTERROGANTE}

¿Qué condiciones metodológicas y de Ingeniería integran un sistema de Gestión de Conocimiento de Apoyo a la Decisión que dote de inteligencia a las organizaciones de producción de bienes y servicios?

Para abordar el problema objeto de estudio se esquematiza y se muestra el diagrama 2 , se parte de la justificación entorno a la necesidad de crear una metodología para proveer de inteligencia a la organización.
Se debe entender entonces que es inteligencia organizacional y cuál es el propósito de proveer de una mayor inteligencia a una organización de bienes y servicios, posteriormente es necesario establecer un modelo de medición de la inteligencia organizacional, identificar los elementos y componentes que la constituyen, que estrategias se pueden plantear para potenciarla, y con qué herramientas, para lo cual es necesario identificar metodologías, métodos y modelos que se utilizan para implementar inteligencia a las organizaciones, determinando que componentes pueden hacer falta para el mejoramiento y formulación de una nueva metodología propósito de la presente investigación.

\section{HIPÓTESIS}

Con la Integración Logística de Conocimiento, que se conFigura como la unión sinérgica de un sistema de soporte a la decisión (DSS) y la gestión del flujo de conocimiento; se dota de inteligencia a las organizaciones de producción de bienes y servicios, con capacidad para actuar exitosamente frente a procesos de toma de decisiones que no son abordados con las teorías y enfoques tradicionales, como se muestra en la ecuación (1).

$f(I 0)=g(D S S) U^{\wedge}((I L C)) h(G C)$

f(IO): Inteligencia Organizacional g(DSS): Función de sistema de apoyo a la decisión

h(GC): Función de gestión de conocimiento $U^{\wedge}(($ ILC) ): Operador de integración logístico de conocimiento que despliega el conocimiento con el uso de herramientas y técnicas de apoyo a la decisión.

\section{MARCO CONCEPTUAL}

Existe una tendencia a considerar la inteligencia organizacional como sinónimo de la inte- 
ligencia de negocios, pero el desarrollo conceptual acerca de la inteligencia de negocios es muy amplio y los aportes son considerables, por lo que existe una gran variedad de información sobre la temática, los principales enfoques están orientados a la desarrollar software y herramientas computacionales para tratar datos que constituyan de alguna manera información base para la toma de decisiones en función del entendimiento y comportamiento de los mercados. Ahora bien el alcance de la inteligencia organizacional es mucho más amplio, la cual abarca todas las actividades orientadas a la potenciación de las capacidades individuales, y colectivas para tomar decisiones oportunas y propiciando la capacidad para adaptarse al cambio.

Con un enfoque integral en donde se gestione el conocimiento desde la formación y capacitación de los individuos que realizan las diferentes actividades en las empresas, para que actúen eficiente y efectivamente, con coordinación y dirigidas al logro de los objetivos estratégicos proyectados hacia el desarrollo y permanencia de las organizaciones.

Es por tanto, fundamental desarrollar una metodología que proveer de inteligencia a las organizaciones en diferentes dimensiones a partir del desarrollo y potenciación de las capacidades individuales y colectivas para posteriormente desplegarla a niveles funcionales y a nivel estratégico, táctico y operativo en la organización y a lo largo de la cadena de abastecimiento. Es función de la inteligencia organizacional la de gestionar las organizaciones a partir del conocimiento en función de su filosofía, su cultura y dinámica al cambio de tendencias que dicta el orden económico y social.

Para proveer de esta inteligencia entonces es necesario construir un sistema de gestión de conocimiento soporte a la decisión con un enfoque integral y dinámico que involucre la participación individual, colectiva y organizacional con capacidad para formular modelos, y usar herramientas y métodos de carácter cuantitativo y cualitativo.

Son características de las organizaciones inteligentes: la capacidad de interactuar con el entorno, lo que implica la coordinación entre las actividades entre los otros eslabones de la cadena, tanto de los proveedores, así como el de los clientes y distribuidores de sus bienes y servicios, su capacidad para adaptarse a las políticas de preservación del medio ambiente, la capacidad para generar, diseñar y gestionar nuevos productos y servicios a partir de la potenciación de los individuos y lo coordinación en equipos de trabajo y la capacidad para tomar decisiones en los procesos de planeación, programación y control de las actividades a nivel funcional, y general.

Uno de los elementos que se debe construir es el sistema de apoyo a la decisión que se conFigura con un conjunto de modelos que se utilizan para clarificar y obtener información para la toma de decisiones eficiente y efectiva en ambientes complejos. Es así como los modelos matemáticos, las herramientas de optimización y las técnicas y herramientas de carácter cuantitativas proporcionan las bases para que el sistema obre en forma inteligente.

Atendiendo lo anterior, la estrategia para construir el marco teórico del proyecto de investigación en Inteligencia Organizacional tiene como componentes los que se observan en la Figura:

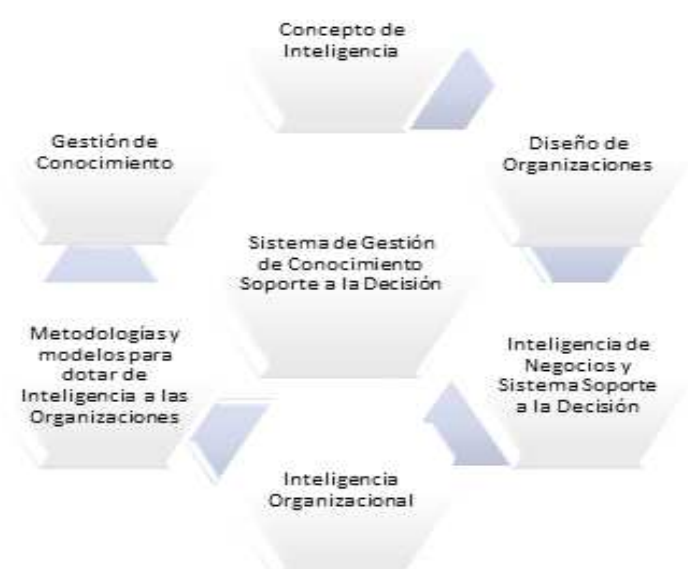

Figura 3.Estrategia para construir el Marco teórico 


\section{a. Concepto de inteligencia}

Para abordar el proceso de investigación conviene definir en principio el concepto de inteligencia como un rasgo propio del ser humano, para posteriormente desplegarlo hacia la concepción de inteligencia de una organización compuesta por organizaciones más pequeñas, dispuestas en funciones o subsistemas, que a su vez se componen de unidades aún más pequeñas, compuestas por seres humanos, lo cual implica por observación que la inteligencia se puede tratar como un sistema fractal, en donde los rasgos individuales se transfieren a los entes organizacionales de nivel superior.

El término inteligencia (del latín intelligentia), que a su vez deriva de inteligere. Esta es una palabra compuesta por otros dos términos: intus ("entre") y legere ("escoger"). Por lo tanto, el origen etimológico del concepto de inteligencia hace referencia a quien sabe elegir: la inteligencia posibilita la selección de las alternativas más convenientes para la resolución de un problema. De acuerdo a lo descrito en la etimología, un individuo es inteligente cuando es capaz de escoger la mejor opción entre las posibilidades que se presentan a su alcance para resolver un problema. [1]

La inteligencia entonces es la capacidad de entender, asimilar, elaborar información y utilizarla para resolver problemas. El Diccionario de la lengua española de la Real Academia Española define la inteligencia, entre otras acepciones como la «capacidad para entender o comprender» y como la «capacidad para resolver problemas». [1] La inteligencia parece estar ligada a otras funciones mentales como la percepción, o capacidad de recibir información, y la memoria, o capacidad de almacenarla. [2] El concepto de "inteligencia" es una tentativa de aclarar y organizar un conjunto complejo de fenómenos. [4]

Definición general del Mainstream Science on Intelligence, definición que fue suscrita por cincuenta y dos investigadores en 1994: [5]

Para poder desplegar las capacidades de inteligencia individual hacia la inteligencia organiza- cional conviene revisar los conceptos acerca de las diferentes teorías acerca de la inteligencia Teoría de la inteligencia Emocional propuesta por Goleman. y la concepción multidimensional de la Inteligencia, y desde un marco también contextual es la propuesta de Inteligencias Múltiples de Howard Gardner [6]

b. Diseño de organizaciones.

Un factor a tener en cuenta en el propósito de proveer a las organizaciones de producción de bienes y servicios es lo relacionado a su estructura organizacional y de la filosofía de gestión de su aparato productivo en por tanto, importante determinar los elementos y principios que conducen el que hacer de las actuar de las organizaciones ya sea, orientadas como sistemas tipo Push o tipo Pull tales como ERP, Lean Manufacturing, TOC o CONWIP.

Estos modelos se basan ya sea en la teoría de los sistemas o en la teoría de la complejidad, pero debido a que hay varios modelos para elegir, juegan un papel más importantes las consideraciones sobre cuál es el más adecuado para una situación específica.

Los modelos de diseño organizacional en general no son parte de las herramientas de los ejecutivos y ofrecen una buena base conceptual para desarrollar un diseño organizacional eficiente.

Los diferentes modelos presentan varias perspectivas de los sistemas de las organizaciones, ninguna de estas perspectivas es la "correcta", la que tienen sentido va depender de las circunstancias, la cultura, el contexto, entre otras cosas. Por los tantos los diseñadores de la organización de una aerolínea podrán usar el modelo de estrella [7] y los diseñadores de la organización de un gran banco podrían favorecerle el modelo de Burke-Litwin. [8]

Enfocar la organización como un sistema es un buen punto de partida para el diseño de la organización.

Es evidente que la dimensión de la inteligencia 
de negocios está reducida al tratamiento de datos para proveer de información a las organizaciones en los proceso de toma de decisiones y no a la de la generación de capacidades para desempeñarse en forma integral de una manera eficiente a nivel individual, funcional y general de las organizaciones.

Para lo cual hay que dotar a la organización de una metodología que promueva la cultura del conocimiento y el aprendizaje organizacional de forma permanente que desplegué los flujos de información, materiales y trabajo en forma eficiente y efectiva a lo largo de la empresa y de la cadena de abastecimiento.

\section{c. Metodologías para proveer de inteligencia a} los negocios

Si bien existen algunas metodologías para evaluar la importancia de la inteligencia de negocios, para implementar programas o ayudas computacionales y metodologías para implementar procesos de análisis de datos, no se ha abordado la solución de problemas de toma de decisiones en varias dimensiones a lo largo de la cadena de suministro y en todos los niveles jerárquicos de decisión.

Es por tanto, importante entender que una metodología para proveer de inteligencia a una organización no se puede limitar al desarrollo de herramientas de soporte al procesamiento de información sino más bien a generar los elementos suficientes para que la organización adquiera conocimiento en todas las dimensiones basado en desarrollo de las capacidades individuales y de grupo a nivel funcional y general de la organización y como eslabón de su cadena de abastecimiento.

Un procedimiento metodológico que aborda toda la organización y también describe los requisitos para una herramienta de soporte que sería adecuada para apoyar la metodología se muestra en [10].

Otras publicaciones están orientadas a procesos de integración y toma de decisiones en tiempo real como [11]
También se encuentran metodologías para medir y evaluar el desempeño Como el propuesto en [12] con redes neuronales que evalúan el éxito o el fracaso en indicadores financieros.

Publicaciones que apuntan a la concientización e implementación de inteligencia de negocios como [13].

En el intento de proveer de inteligencia a las organizaciones se propone el uso de cuadros de mando integral

\section{d. Sistema apoyo a la decisión}

Un DSS puede adoptar muchas formas diferentes. En general, se puede decir que un DSS es un sistema informático utilizado para servir de apoyo, más que automatizar, el proceso de toma de decisiones. La decisión es una elección entre alternativas basadas en estimaciones de los valores de esas alternativas. El apoyo a una decisión significa ayudar a las personas que trabajan solas o en grupo a reunir inteligencia, generar alternativas y tomar decisiones. Apoyar el proceso de toma de decisión implica el apoyo a la estimación, la evaluación y/o la comparación de alternativas. En la práctica, las referencias a DSS suelen ser referencias a aplicaciones informáticas que realizan una función de apoyo. [15]

El término sistema de apoyo a la decisión se ha utilizado de formas muy diversas y se ha definido de diferentes maneras dependiendo del punto de vista del autor [16] Algunas de esas definiciones son:

- Un DSS, en términos muy generales, es "un sistema basado en computador que ayuda en el proceso de toma de decisiones" [17].

- $\quad$ En términos bastante más específicos, un DSS es "un sistema de información basado en un computador interactivo, flexible y adaptable, especialmente desarrollado para apoyar la solución de un problema de gestión no estructurado para mejorar la toma de decisiones. Utiliza datos, proporciona una interfaz amigable y permite la toma de decisiones en el propio análisis de la situación" [18]

Otras definiciones intermedias entre las dos 
anteriores serían:

- Un DSS es un "conjunto de procedimientos basados en modelos para procesar datos y juicios para asistir a un gerente en su toma de decisiones" [19]

- Un DSS "combina recursos intelectuales individuales con las capacidades de un ordenador para mejorar la calidad de las decisiones (son un apoyo informático para los encargados de tomar decisiones sobre problemas semiestructurados)" [20]

- $\quad$ "Sistema extensible capaz de apoyar ad-hoc el análisis de datos y el modelado de decisiones, orientado a la planificación futura y utilizado a intervalos irregulares, no planificados" [21]

- Los DSS son "Sistemas informáticos interactivos que ayudan a los encargados de tomar decisiones utilizando datos y modelos para resolver problemas no estructurados" [22]

- $\quad$ Keen afirma que es imposible dar una definición precisa incluyendo todas las facetas de la DSS ya que "no puede haber una definición de los sistemas de apoyo a la decisión, sino sólo del apoyo a la decisión" [23]

- $\quad$ Para Power el término DSS puede referirse a muchos tipos de sistemas de información que dan soporte a la toma de decisiones. Humorísticamente añade que siempre que un sistema informático no sea un 'sistema para procesamiento de transacciones en linea' (OLTP), alguien tendrá la tentación de llamarlo DSS [24]

\section{e. Concepto de inteligencia organizacional}

El término inteligencia visto psicológicamente "capacidad de adquirir conocimiento o entendimiento y de utilizarlo en situaciones novedosas" se emplea desde finales del siglo XIX. En el ámbito gerencial, debe su origen a las actividades militares en las que se requiere una considerable "inteligencia" para acceder a las fuentes, obtener información sobre el enemigo y entregarla a los mandos que deben tomar las decisiones "los miembros de la "inteligencia" no toman las decisiones por sí mismos.

Es así como surge una acepción diferente de la actividad y del sistema de inteligencia, que no abarca todo lo que el término psicológico comprende.

La inteligencia empresarial cubre perfectamente las expectativas en el entorno económico como herramienta para obtener un gran volumen de información útil a los directivos.

Se cuenta con las siguientes divisiones: Planeación estratégica, Desarrollo organizacional, Ventas, Desarrollo de Líderes, Desarrollo Empresarial, Desarrollo Humano, Administración de la Calidad Total.

Contribuir al incremento de la competitividad de nuestros clientes, a través de la transferencia de tecnología organizacional, con la mejor relación costo/beneficio del mercado". [28]

\section{f. La Creación de Conocimiento Organizacio- nal}

La Creación de Conocimiento Organizacional, según [38] debe entenderse como la capacidad orgánica para generar nuevos conocimientos, difundiéndolos entre los miembros de una organización y materializándolos en productos, servicios y sistemas. Es la clave del proceso a través del cual las firmas innovan.

El resultado de este proceso son un conjunto de conocimiento que circulan por los diferentes niveles organizacionales de conocimiento: individual, grupal y organizacional. Según las consideraciones de autores como [38] y [34] acerca de los tipos de conocimiento se pueden identificar en cada nivel la presencia del conocimiento tácito y el explícito.

El conocimiento tácito es aquel que forma parte de las experiencias de aprendizaje personal de cada individuo y que por tanto resulta sumamente complicado, imposible de estructurar, almacenar en repositorios y distribuir.

Por su parte el conocimiento explícito es aquel que se puede expresar con palabras y números, y comunicarse y compartirse con facilidad en la forma de datos concretos y fórmulas científicas, procedimientos codificados, o principios universales. 
Estos conocimientos son los que apoyan el proceso de la innovación, pero la creación de los mismos está condicionada por las formas de creación y conversión que adopte cada organización. Entre los modelos de creación de conocimiento se encuentran el de [38] y el de [39]. El primero muestra la creación o conversión de conocimiento por medio de los procesos de Socialización, Externalización, Internalización y Combinación, y el segundo muestra la dinámica de creación a partir de las redes sociales y del uso de nuevas tecnologías, así como las ventajas que brinda el conocimiento una vez creado. En la creación o transformación de conocimientos, la información es importante ya que esta constituye la expresión del mismo. Los procesos de transformación de conocimiento (dígase socialización, externalización, internalización y combinación) son proceso informacionales por el uso que hacen de este recurso.

Socialización: los individuos o grupos comparten sus experiencias o conocimientos mediante diversas vías - entre las que sobresalen la observación y el diálogo - que llevan explícito el intercambio de información.

Externalización: se expresan o se documentan los conocimientos que un individuo posee, es decir se explícita ese conocimiento en información.

Combinación: permite crear conocimiento a partir del uso y análisis de información, ya que los individuos interpretan o analizan información que puede o no estar documentada y luego de hacerlo generan un nuevo conocimiento.

Internalización: en este proceso se aprende e interiorizan nuevos conocimientos a partir del análisis de información.

En cada uno de estos procesos la información es imprescindible, en ocasiones esta se presenta mediante palabras, gestos o documentos y contribuye a que los individuos creen sus conocimientos.

De acuerdo con estos conceptos, la Gestión del Conocimiento es un proceso que garantiza y facilita la creación, intercambio y uso de conocimiento al desarrollar acciones que permitan identificar, adquirir, almacenar, intercambiar, aplicar y asegurar el conocimiento en una organización e involucrar este recurso en la innovación y en el logro de altos indicadores de éxito y de mejoras continuas.

g. La Inteligencia Organizacional como modelo integrado de Gestión de Información y del Conocimiento

Como se ha demostrado la Inteligencia Organizacional depende de la información, el conocimiento y el uso que se proporciona a estos recursos en las organizaciones, por lo que la Gestión de Información y del Conocimiento influyen en el óptimo desarrollo de esta capacidad al crear las condiciones para tratar adecuadamente a los mismos.

La importancia e interrelación de la Gestión de Información y del Conocimiento en la IO permite afirmar que la implementación de la misma como capacidad está sustentada en el desarrollo de ambos.

La integración mostrada anteriormente es la que propicia que la organización pueda efectuar una toma de decisiones efectiva y que se adapte a los cambios de su ambiente.

La Gestión de Información permite a la organización administrar eficiente y eficazmente los recursos informativos por lo que contribuye a un mejor desarrollo de cada proceso de la IO.

La Gestión del Conocimiento permite a la organización un adecuado uso de sus recursos para desarrollar los procesos de conocimiento por lo que contribuye a un óptimo desarrollo de cada proceso de la Inteligencia.

La implementación de la IO como capacidad está sustentado en el desarrollo de la Gestión de Información y del Conocimiento, lo que permite crear las condiciones organizacionales para un acertado uso de estos recursos.

En la literatura especializada se usan indistintamente los términos Inteligencia Empresarial, Corporativa u Organizacional, para definir esta capacidad. 


\section{Referencias}

[1] «Inteligencia,» de Diccionario de la lengua española (22. ${ }^{\text {a }}$ edición), Real Academia Española, 2001.

[2] de Manual de psiquiatría médica ( $2^{\mathrm{a}}$ ed.), España, Elsavier, 2005.

[3] P. E. Vernon, Intelligence and attainment tests., Oxford, England: Univer, London Press. 207 pp. , 1960.

[4] U. Neisser, G. Boodoo, T. J. Bouchard Jr., A. W. Boykin, N. Brody, S. J. Ceci, D. F. Halpern, J. C. Loehlin, R. Perloff, R. J. Sternberg y S. Urbina, «Intelligence: Knowns and unknowns.,» American Psychologist APA Task Force Report, pp. Vol 51(2), 77-101., Feb 1996.

[5] L. Gottfredson y D. H. Saklofske, «Intelligence: Foundations and issues in assessment. „» Canadian Psychology/ Psychologie canadienne, Vol 50(3), pp. 183-195., Aug 2009.

[6] H. Gardner, Inteligencias múltiples, Paidos, ISBN 950-12-5012-1., 2003.

[7] J. Galbraith, Designing Organizations. San Francisco, California, EUA: JosseyBass, San Francisco, California, : EUA: Jossey-Bass, 1995.

[8] W. \&. L. G. H. BURKE, «A causal model of organizational performance and change.,» Journal of management , pp. 18 (3), 523-545, 1992.

[9] B. T. Alexander Mikroyannidis $\square$, «Ontology management and evolution for business intelligence,» International Journal of Information Management , p. 30 559-566, 2010.

[10] K. M. Bajec Marko, «A methodology and tool support for managing business rules in organisations,» Information Systems 30, p. 423-443, 2005.

[11] M. F. Tanko Ishaya, «A service oriented approach to Business Intelligence in Telecoms industry,» Telematics and Informatics, p. 29 273-285, 2012.

[12] S. C. C. K. B.S. Ahna, «The integrated methodology of rough set theory and artificial neural,» Expert Systems with
Applications , p. 18 65-74, 2000.

[13] S. O.-a. Kamel Rouibah, «PUZZLE: a concept and prototype for linking business Intelligence to business strategy,» Journal of StrategyInformation Systems, pp. 11 133-152, 2002.

[14] Metodología para el Diseñoe Implantación de un Sistema de BI, tecnologia y decisiones , 2013.

[15] S. L. Alter, Decision support systems: current practice and continuing challenges., Reading, Mass., AddisonWesley Pub., 1980.

[16] M. J. a. R. R. F. Druzdzel, Decision Support Systems., Encyclopedia of Library and Information Science. A. Kent, Marcel Dekker, Inc., 1999.

[17] P. N. Finlay, Introducing decision support systems., Oxford, UK Cambridge, Mass.: NCC Blackwell; Blackwell Publishers., 1994.

[18] E. Turban, Decision support and expert systems: management support systems., Englewood Cliffs, N.J., Prentice Hall. , 1995.

[19] J. Little, «"Models and Managers:The Concept of a Decision Calculus.",» Management Science, pp. Vol.16,NO.8, 1970, April.

[20] P. G. W. Keen, Decision support systems: a research perspective. Decision support systems : issues and challenges., G. Fick and R. H. Sprague. Oxford ; New York, Pergamon Press., 1980.

[21] Moore, J.H., M.G.Chang., «"Design of Decision Support Systems.",» Data Base,Vol.12, Nos.1 and 2., 1980.

[22] Sprague, R. H. and E. D. Carlson, Building effective decision support systems., Englewood Cliffs, N.J., Prentice-Hall., 1982.

[23] Keen, P. G. W., Decision support systems: an organizational perspective., Reading, Mass., Addison-Wesley Pub. Co., 1978.

[24] D. J. Power, «What is a DSS?,» The OnLine Executive Journal for Data-Intensive Decision Support 1(3)., 1997.

[25] D. A. Power, «Brief History of Decision Support Systems,» DSSResources.COM World Wide Web, version 2.8, May 31, 
2003.

[26] Richelson, The U.S. Intelligence Community., New York: Ballinger;: p. 1., 1989.

[27] T. U.S, Intelligence Community. 4th., ed. Boulder: Westview Press; 1999.

[28] P. Senge, La Quinta Disciplina, Garnica, 1994.

[29] Y. R. Cruz y E. G. Domínguez, «La inteligencia organizacional: necesario enfoque de gestión de información y del conocimiento,» Ciência da Informação , p. Ci. Inf. vol.36 no.3 Brasília, Sept./Dec. 2007.

[30] A. Wilensky, Claves de La Estrategia Competitiva, Buenos Aires, Argentina: OSDE, 1997.

[31] Haeckel, S. H. and Nolan, K. L., «The role of technology in an information age: transforming symbols into action. In The Institute for Information Studies. The knowledge Economy: The nature of information in the 21st Century. Queenstown: The Aspen I,» 1993.

[32] C. W. Choo, «Information management for the intelligent organization: the art of scanning the enviroment,» vol. $325 \mathrm{p}$., 2002.

[33] W., Halal, «Organizational Intelligence: What is it and how can managers use it?,» [En línea]. Available: Diponible en: http:// www.strategy-business.com/briefs/97413 html..

[34] C. W. Choo, «Information management for the intelligent organization: the art of scanning the enviroment.,» New Jersey: American Society for the Information Science and Tecnology, p. 325 p., 2002.

[35] D. A. Garvin, «Building a learning organization.,» Harvard Business Review. July / August., 1993.

[36] NuñezPaula,I., Gestion delConocimiento. Conceptos, aplicaciones y experiencias. En: Seminario Iberoamericano sobre tendencias modernas en Gerencia de la Ciencia y la Innovacion Tecnologica., La Habana: 2002. Editorial Academia, p. 185., 2002.

[37] K. E. Weick, «Weick, K. E. (1995). Sensemaking in Organizations.,»
[38]

Thousand Oaks, CA: Sage., 1995.

Nonaka, I. and Takeuchi, H., The knowledgecreatingcompany: how japanese companies create the dynamics of innovation., New York:: Oxford University Press., 1995.

[39] J. Carrión, «"Modelo general para la creación de conocimiento". Fundación iberoamericana del conocimiento.,» 2002. [En línea]. Available: www. gestiondelconocimiento.com.

[40] Davenport, Thomas y Prusak,, Laurance Working Knowledge., Boston: Harvard Business School Press., 1998.

[41] Wiig, Karl M., «Knowledge Management: An Emerging Discipline Rooted in a Long History Knowledge Research Institute, Inc,» de Knowledge Management, Edited by Daniele Chauvel \& Charles Despres Scheduled for publication Fall, , 1999.

[42] G. Ponjuán Dante, Gestión de Información en las Organizaciones: Principios, conceptos y aplicaciones., Santiago de Chile:: CECAPI, 1998.

[43] D. Goleman, Inteligencia emocional., Barcelona.: Kairos., 1996.

[44] R. J. Sternberg, A Triarchic Theory of Intelligence., Cambridge: University Press., 1985.

[45] A. Pueyo, Manual de Psicología Diferencial., España: Mc Hill. pp. 4., 1996.

[46] M. J. S. R. M. Ghazanfari, «A tool to evaluate the business intelligence of enterprise systems,» Scientia Iranica E , pp. 18 (6), 1579-1590, 2011.

[47] P. Haettenschwiler, " Neues anwenderfreundliches Konzept der Entscheidungsunterstützung.,» Gutes Entscheiden in Wirtschaft, Politik und Gesellschaft. Zurich, vdf Hochschulverlag AG: 189-208., 1999.

[48] D. J. Power, Decision support systems: concepts and resources for managers., Westport, Conn., Quorum Books., 2002.

[49] A. Gachet, Building Model-Driven Decision Support Systems with Dicodess., Zurich, VDF., 2004.

[50] P. Stanhope, Get in the Groove: building 
tools and peer-to-peer solutions with the Groove platform., New York: Hungry Minds, 2002.

[51] C.M.P.D. Haag, Management Information Systems: For The Information Age., McGraw-Hill Ryerson Limited: 136-140. , 2000.

[52] P. Haettenschwiler, Neues anwenderfreundliches Konzept der Entscheidungsunterstützung., Zurich, vdf Hochschulverlag AG: 189-208.: Gutes Entscheiden in Wirtschaft, Politik und Gesellschaft., 1999.

[53] G. M. Marakas, Decision support systems in the twenty-first century., Upper Saddle River, N.J.: Prentice Hall., 1999.

[54] H. T. Nonaka I, The knowledge creating Company., Nueva York:: Oxford
University Press; 1995.

[55] H. T. Nonaka I, The knowledge creating Company., Nueva York: : Oxford University Press;, 1995.

[56] C. CW., The knowing organization: how organizations use information to construct meaning, create knowledge and make decisions., New York:: Oxford University Press; 1998.

[57] C. CW., Information management for the intelligent organization: the art of scanning the environment. 2nd. ed, Medford: Information Today; , 1998.

[58] D. J. Philip H, Intelligence, Information Technology and Information Warfare., Kuala Lumpur:: University of Malaya; 2002. 